\title{
A Kernel to Exploit Informative Missingness in Multivariate Time Series from EHRs
}

\author{
Karl Øyvind Mikalsen ${ }^{* 1,2}$, Cristina Soguero-Ruiz ${ }^{1,3}$, Robert Jenssen ${ }^{1}$ \\ ${ }^{1}$ Dept. of Physics and Technology, UiT The Arctic University of Norway, NO-9037 Troms $\emptyset$, Norway \\ ${ }^{2}$ University Hospital of North-Norway, ${ }^{3}$ Rey Juan Carlos University, Fuenlabrada, Spain \\ karl.o.mikalsen@uit.no, cristina.soguero@urjc.es, robert.jenssen@uit.no
}

\begin{abstract}
A large fraction of the electronic health records (EHRs) consists of clinical measurements collected over time, such as lab tests and vital signs, which provide important information about a patient's health status. These sequences of clinical measurements are naturally represented as time series, characterized by multiple variables and large amounts of missing data, which complicate the analysis. In this work, we propose a novel kernel which is capable of exploiting both the information from the observed values as well the information hidden in the missing patterns in multivariate time series (MTS) originating e.g. from EHRs. The kernel, called $\mathrm{TCK}_{I M}$, is designed using an ensemble learning strategy in which the base models are novel mixed mode Bayesian mixture models which can effectively exploit informative missingness without having to resort to imputation methods. Moreover, the ensemble approach ensures robustness to hyperparameters and therefore $\mathrm{TCK}_{I M}$ is particularly well suited if there is a lack of labels - a known challenge in medical applications. Experiments on three real-world clinical datasets demonstrate the effectiveness of the proposed kernel.
\end{abstract}

\section{Introduction}

The widespread growth of electronic health records (EHRs) has generated vast amounts of clinical data. Normally, an encounter-based patient EHR is longitudinal and contains clinical notes, diagnosis codes, medications, laboratory tests and vital signs, etc., which can be represented as multivariate time series (MTS). As a consequence, EHRs contain valuable information about the clinical observations depicting both patients health and care provided by physicians. However, the longitudinal and heterogeneous data sources make EHR analysis difficult from a computational perspective. In addition, the EHRs are often subject to a lack of completeness, implying that the MTS extracted from EHRs often contain massive missing data (Sharafoddini and others 2019). Missing data might, however, occur for different reasons. It could be that the physician orders lab tests, but because of an error some results are not recorded. On the other hand, it can also happen that the physician decides to not order lab tests because he thinks the patient is in good shape. In the

\footnotetext{
${ }^{*}$ Corresponding author. https://machine-learning.uit.no/
}

first case, the missingness is ignorable, whereas in the latter case, the missing values and patterns potentially can contain rich information about the patient's diseases and clinical outcomes. Efficient data-driven systems aiming to extract knowledge, perform predictive modeling, etc., must be capable of capturing this information.

Traditionally, missingness mechanisms have been divided into missing completely at random (MCAR), missing at random (MAR) and missing not at random (MNAR). The main difference between these mechanisms consists in whether the missingness is ignorable (MCAR and MAR) or nonignorable (MNAR) (Donders and others 2006; Rubin 1976). This traditional description of missingness mechanisms is, however, not always sufficient in medical applications as the missing patterns might be correlated with additional variables, such as e.g. a disease. This means that the distribution of the missing patterns for patients with a particular disease might be different than the corresponding distribution for patients without the disease, i.e. the missingness is informative (Ghorbani and Zou 2018; Wells et al. 2013).

Several methods have been proposed to handle missing data in MTS (Little and Rubin 2014, Schafer and Graham 2002). A simple approach is to create a complete dataset by discarding the MTS with missing data. Alternatively, one can do simple imputation of the missing values, e.g. using the last observation carried forward scheme (impute the last non-missing value for the following missing values) (Shao and Zhong 2003), zero-value imputation (replace missing values with zeros) or mean-value imputation (missing values are replaced with the mean of the observed data) (Zhang 2016). A common limitation of these approaches is that they lead to additional bias, loss of precision, and they ignore uncertainty associated with the missing values (Donders and others 2006). This problem is to some extent solved via multiple imputation methods, i.e. by creating multiple complete datasets using single imputation independently each time. Then, by training a classifier using an ensemble learning strategy, one can improve the performance compared to simple imputation. However, this imputation procedure is an adhoc solution as it is performed independently of the rest of the analysis and it ignores the potential predictive value of the missing patterns (Ma and Chen 2018). 
Due to the limitations of imputation methods, several research efforts have been devoted over the last years to deal with missing data in MTS using alternative strategies (Bianchi et al. 2018; Bianchi and others 2019, Che and et al. 2018; Ghassemi and others 2015; Li and $\mathrm{Xu} 2019$; Lipton, Kale, and Wetzel 2016, Mikalsen et al. 2016, Shukla and Marlin 2019). Prominent examples are kernels, i.e. positive semi-definite time series similarity measures, such as the learned pattern similarity (LPS) (Baydogan and Runger 2016) and the time series cluster kernel (TCK) (Mikalsen and others 2018) that can naturally deal with missing data. The former generalizes autoregressive models to local autopatterns, which capture the local dependency structure in the time series, and uses an ensemble learning (random forest) strategy in which a bag-of-words representation is created from the output of the leaf-nodes for each tree. TCK is also based on an ensemble learning approach and shares many properties with LPS. It is designed using an ensemble learning approach in which Bayesian mixture models form the base models. However, while LPS exploits the inherent missing data handling abilities of decision trees, TCK is a likelihood-based approach in which the incomplete dataset is analysed using maximum a posteriori expectationmaximization. An advantage of these methods, compared to e.g. multiple imputation that requires a careful selection of imputation model and parameters (Schafer and Graham 2002), is that the missing data are handled automatically and no additional tasks are left to the designer. Additionally, since the methods are based on ensemble learning, they are robust to hyperparameter choices. In particular, these properties are important in unsupervised settings, which frequently occur in medical applications where manual label annotation of large datasets often is not feasible (Halpern and others 2016, Mikalsen et al. 2017).

A shortcoming of these kernel methods is, however, that they cannot exploit informative missing patterns, which frequently occur in medical MTS, and unbiased predictions are only guaranteed for ignorable missingness as MAR is an underlying assumption. Recently, several studies have focused on modeling the informative or nonignorable missigness by analyzing the observed values as well as the indicators of missingness, concluding that the missing patterns can add more insights beyond the observed values (Agniel and others 2018, Che and et al. 2018, Lipton, Kale, and Wetzel 2016; Sharafoddini and others 2019). In this work, we present a novel time series cluster kernel, $\mathrm{TCK}_{I M}$, that also represents the missing patterns using binary indicator time series. By doing so, we obtain MTS consisting of both continuous and discrete attributes. However, we do not only concatenate the binary MTS to the real-valued MTS and analyse these data in a naive way. Instead, we take a statistically principled Bayesian approach (Little and Rubin 2014; Ma and Chen 2018) and model the missingness mechanism more rigorously by introducing novel mixed mode Bayesian mixture models, which can effectively exploit information provided by the missing patterns as well as the temporal dependencies in the observed MTS. The mixed mode Bayesian mixture models are then used as base models in an ensemble learning strategy to form the $\mathrm{TCK}_{I M}$ kernel. Experiments on three real-world datasets of patients described by longitudinal EHR data, demonstrate the effectiveness of the proposed method.

\section{Time series cluster kernel to exploit informative missingness}

Here we present the proposed $\mathrm{TCK}_{I M}$ kernel. The kernel is learned using an ensemble learning strategy, i.e. by training individual base models which are combined into a composite kernel in the end. As base model we introduce a novel mixed mode Bayesian mixture model. Before we provide the details of this method, we describe the notation used throughout the paper.

Notation We define a multivariate time series (MTS) $X$ as a finite combination of univariate time series (UTS) $x_{v}$ of length $T$, i.e. $X=\left\{x_{v} \in \mathbb{R}^{T} \mid v=1,2, \ldots, V\right\}$. The dimensionality of the MTS $X$ is the same as the number of UTS, $V$, and the length of $X$ is the same as the length $T$ of the UTS $x_{v}$. A $V$-dimensional MTS, $X$, of length $T$ can be represented as a matrix in $\mathbb{R}^{V \times T}$. Given a dataset of $N$ MTS, we denote $X^{(n)}$ as the $n$-th MTS. In a dataset of $N$ incompletely observed MTS, the $n$-th MTS is denoted by the pair $U^{(n)}=\left(X^{(n)}, R^{(n)}\right)$, where $R^{(n)}$ is a binary MTS with entry $r_{v}^{(n)}(t)=0$ if the realization $x_{v}^{(n)}(t)$ is missing and $r_{v}^{(n)}(t)=1$ if it is observed.

Mixed mode Bayesian mixture model Let $U=(X, R)$ be a MTS generated from two modes, where $X$ is a $\mathrm{V}$-variate real-valued MTS $\left(X \in \mathbb{R}^{V \times T}\right)$ and $R$ is a V-variate binary MTS $\left(R \in\{0,1\}^{V \times T}\right)$. In the mixture model it is assumed that $U$ is generated from a finite mixture density

$$
p_{u}(U \mid \Phi, \Theta)=\sum_{g=1}^{G} \theta_{g} p_{u_{g}}\left(U \mid \phi_{g}\right),
$$

where $G$ is the number of components, $p_{u_{g}}$ is the density of the components parametrized by $\Phi=\left(\phi_{1}, \ldots, \phi_{G}\right)$, and $\Theta=\left(\theta_{1}, \ldots, \theta_{G}\right)$ are the mixing coefficients, $0 \leq \theta_{g} \leq 1$ and $\sum_{g=1}^{G} \theta_{g}=1$. We formulate the mixture model in terms of a latent random variable $Z$, described via the onehot vector $Z=\left(Z_{1}, \ldots, Z_{G}\right)$ with marginal distribution given by $p_{z}(Z \mid \Theta)=\prod_{g=1}^{G} \theta_{g}^{Z_{g}}$. The latent variable $Z$ describes which cluster component the MTS $U$ belongs to, i.e. $Z_{g}=1$ if $U$ belongs to cluster component $g$ and $Z_{g}=0$ otherwise. The conditional is given by $p_{u \mid z}(U \mid Z, \Phi)=$ $\prod_{g=1}^{G} p_{u_{g}}\left(U \mid \phi_{g}\right)^{Z_{g}}$, and therefore it follows that the joint distribution is given by

$$
\begin{aligned}
p_{u, z}(U, Z \mid \Phi, \Theta) & =p_{u \mid z}(U \mid Z, \Phi) p_{z}(Z \mid \Theta) \\
= & \prod_{g=1}^{G}\left[p_{u_{g}}\left(U \mid \phi_{g}\right) \theta_{g}\right]^{Z_{g}} .
\end{aligned}
$$

We further assume that the parameters of each component are given by $\phi_{g}=\left(\mu_{g}, \Sigma_{g}, \beta_{g}\right)$ and

$$
p_{u_{g}}\left(U \mid \phi_{g}\right)=p_{x \mid r}\left(X \mid R, \mu_{g}, \Sigma_{g}\right) p_{r}\left(R \mid \beta_{g}\right),
$$

where $p_{x \mid r}$ is a density function given by

$p_{x \mid r}\left(X \mid R, \mu_{g}, \Sigma_{g}\right)=\prod_{v=1}^{V} \prod_{t=1}^{T} \mathcal{N}\left(x_{v}(t) \mid \mu_{g v}(t), \sigma_{g v}\right)^{r_{v}(t)}$, 
where $\mu_{g}=\left\{\mu_{g v} \in \mathbb{R}^{T} \mid v=1, \ldots, V\right\}$ is a time-dependent mean, and $\Sigma_{g}=\operatorname{diag}\left\{\sigma_{g 1}^{2}, \ldots, \sigma_{g V}^{2}\right\}$ is a diagonal covariance matrix in which $\sigma_{g v}^{2}$ is the variance of attribute $v$. Hence, the covariance is assumed to be constant over time. $p_{r}$ is a probability mass given by

$$
p_{r}\left(R \mid \beta_{g}\right)=\prod_{v=1}^{V} \prod_{t=1}^{T} \beta_{g v t}^{r_{v}(t)}\left(1-\beta_{g v t}\right)^{1-r_{v}(t)},
$$

where $\beta_{g v t} \in[0,1]$. The idea with this formulation is to use the Bernoulli term $p_{r}$ to capture information from the missing patterns and $p_{x \mid r}$ to capture the information from the observed data.

Using Bayes' theorem we compute the conditional probability of $Z$ given $U, P\left(Z_{g}=1 \mid U, \Phi, \Theta\right)$,

$$
\pi_{g}=\frac{\theta_{g} p_{x \mid r}\left(X \mid R, \mu_{g}, \Sigma_{g}\right) p_{r}\left(R \mid \beta_{g}\right)}{\sum_{g=1}^{G} \theta_{g} p_{x \mid r}\left(X \mid R, \mu_{g}, \Sigma_{g}\right) p_{r}\left(R \mid \beta_{g}\right)} .
$$

To improve the capability of handling missing data, a Bayesian extension is introduced where informative priors are put over the parameters of the normal distribution as well as the Bernoulli distribution. This enforces that the cluster representatives become smooth over time even in the presence of large amounts of missing data and that the parameters of clusters with few MTS are similar to the overall mean. Towards this end, a kernel-based Gaussian prior is defined for the mean,

$$
p_{\mu}\left(\mu_{g v}\right)=\mathcal{N}\left(\mu_{g v} \mid m_{v}, S_{v}\right),
$$

where $m_{v}$ are the empirical means and $S_{v}=s_{v} \mathcal{K}$, are the prior covariance matrices. $s_{v}$ are empirical standard deviations and $\mathcal{K}$ is a kernel matrix, whose elements are $\mathcal{K}_{t t^{\prime}}=$ $b_{0} \exp \left(-a_{0}\left(t-t^{\prime}\right)^{2}\right), \quad t, t^{\prime}=1, \ldots, T$, with $a_{0}, b_{0}$ being user-defined hyperparameters. For the standard deviation $\sigma_{g v}$, an inverse Gamma distribution prior is introduced

$$
p_{\sigma}\left(\sigma_{g v}\right) \propto \sigma_{g v}^{-N_{0}} e^{-N_{0} s_{v} / 2 \sigma_{g v}^{2}},
$$

where $N_{0}$ is a hyperparameter. Further, we put a Beta distribution prior on $\beta_{\text {gvt }}$

$$
p_{\beta}\left(\beta_{g v t}\right) \propto \beta_{g v t}^{c_{0}-1}\left(1-\beta_{g v t}\right)^{d_{0}-1} .
$$

where $c_{0}, d_{0}$ are hyperparameters. We let $\Omega=$ $\left\{a_{0}, b_{0}, c_{0}, d_{0}, N_{0}\right\}$ denote the set all of hyperparameters.

Given a dataset $\left\{U^{(n)}\right\}_{n=1}^{N}$, we estimate the parameters $\{\Phi, \Theta\}$ using maximum a posteriori expectation maximization (MAP-EM) (Dempster, Laird, and Rubin 1977). The Qfunction is computed as follows

$$
\begin{aligned}
Q= & \mathbb{E}_{Z \mid U, \Theta, \Phi}\left[\log \left(p_{u, z}(U, Z \mid \Phi, \Theta) p(\Phi)\right)\right] \\
= & \log p(\Phi)+\sum_{n, g} \log \left[p_{u}\left(U^{(n)} \mid Z_{g}^{(n)}=1, \Phi\right) p\left(Z_{g}^{(n)} \mid \Theta\right)\right] \pi_{g}^{(n)} \\
= & \sum_{g} \log \left(p_{\mu}\left(\mu_{g}\right) p_{\sigma}\left(\sigma_{g}\right) p_{\beta}\left(\beta_{g}\right)\right) \\
& +\sum_{n, g} \log \left[p_{x \mid r}\left(X^{(n)} \mid R^{(n)}, \mu_{g}, \Sigma_{g}\right) p_{r}\left(R^{(n)} \mid \beta_{g}\right) \theta_{g}\right] \pi_{g}^{(n)},
\end{aligned}
$$

where $p_{x \mid r}$ is given by Eq. (4), $p_{r}$ by Eq. (5), $p_{\mu}$ by Eq. (7), $p_{\sigma}$ by Eq. (8) and $p_{\beta}$ by Eq. (9).

The E-step in MAP-EM is the same as in maximum likelihood EM and consists in updating the posterior (Eq. (6)) using the current parameter estimates, whereas the M-step consists in maximizing the Q-function (Eq. 10) wrt. the parameters $\{\Phi, \Theta\}$. I.e.,

$$
\left\{\Phi^{(m+1)}, \Theta^{(m+1)}\right\}=\arg \max _{\{\Phi, \Theta\}} Q\left(\Phi, \Theta \mid \Phi^{(m)}, \Theta^{(m)}\right)
$$

Computing the derivatives of $Q$ with respect to the parameters $\theta_{g}, \mu_{g}, \sigma_{g}$ and $\beta_{g}$ leads to Alg. 1 .

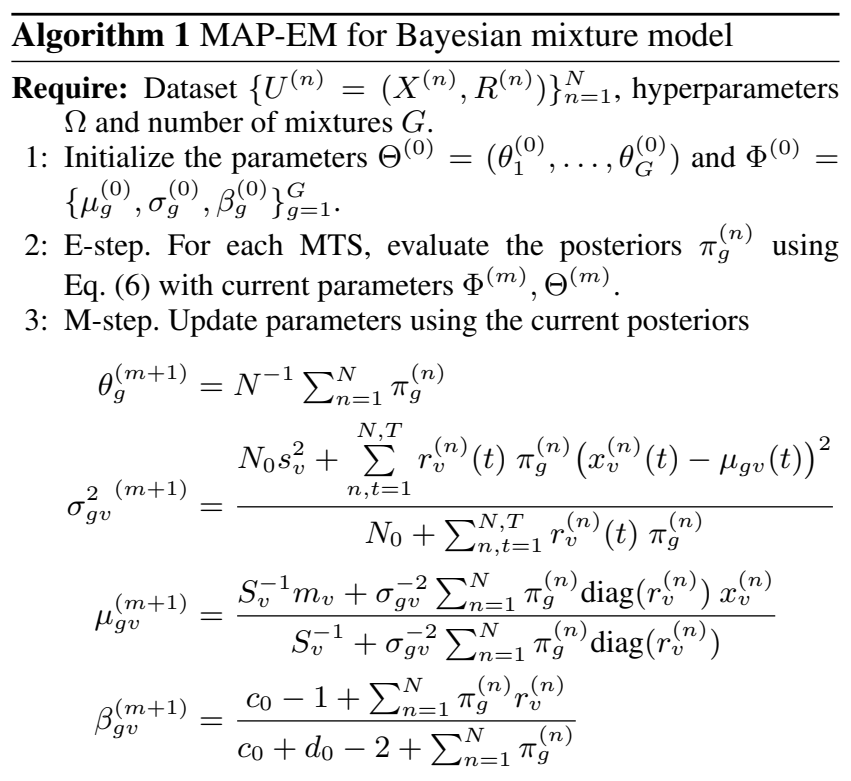

4: Repeat step 2-3 until convergence.

Ensure: Posteriors $\Pi^{(n)} \equiv\left(\pi_{1}^{(n)}, . ., \pi_{G}^{(n)}\right)$ and parameters $\Theta, \Phi$.

The TCK $_{I M}$ kernel To compute the $\mathrm{TCK}_{I M}$ kernel, we use the mixed mode Bayesian mixture model, described above, as the base model in an ensemble approach. Key to ensure that $\mathrm{TCK}_{I M}$ will have statistical advantages (lower variance), computational advantages (less sensitive to local optima) as well as representational advantages (increased expressiveness) compared to the individual base models, is diversity and accuracy (Dietterich 2000, Hansen and Salamon 1990). In general, this means that the base models should not do the same type of errors and each base model has to perform better than random guessing. Hence, to ensure diversity, we integrate multiple outcomes of the base model as it is trained under different, randomly chosen, settings (hyperparameters, initialization, subsampling). In more detail, the number of cluster components for the base models is sampled from a set of integers $\mathcal{I}_{C}=\{I, \ldots, I+$ $C\}$. For each number of cluster components $q_{2} \in \mathcal{I}_{C}$, we apply $Q$ different random initial conditions and sample hyperparameters uniformly as follows: $a_{0} \in[0.001,1]$, $b_{0} \in[0.005,0.2], n_{0} \in[0.001,0.2], c_{0}, d_{0} \in[0.1 / N, 2 / N]$. We let $\mathcal{Q}=\left\{q=\left(q_{1}, q_{2}\right) \mid q_{1}=1, \ldots Q, q_{2} \in \mathcal{I}_{C}\right\}$ be the index set keeping track of initial conditions and hyperparameters $\left(q_{1}\right)$ as well as the number of components $\left(q_{2}\right)$. Each base model $q$ is trained on a random subset of MTS $\left\{\left(X^{(n)}, R^{(n)}\right)\right\}_{n \in \eta(q)}$. To further increase the diversity, for 

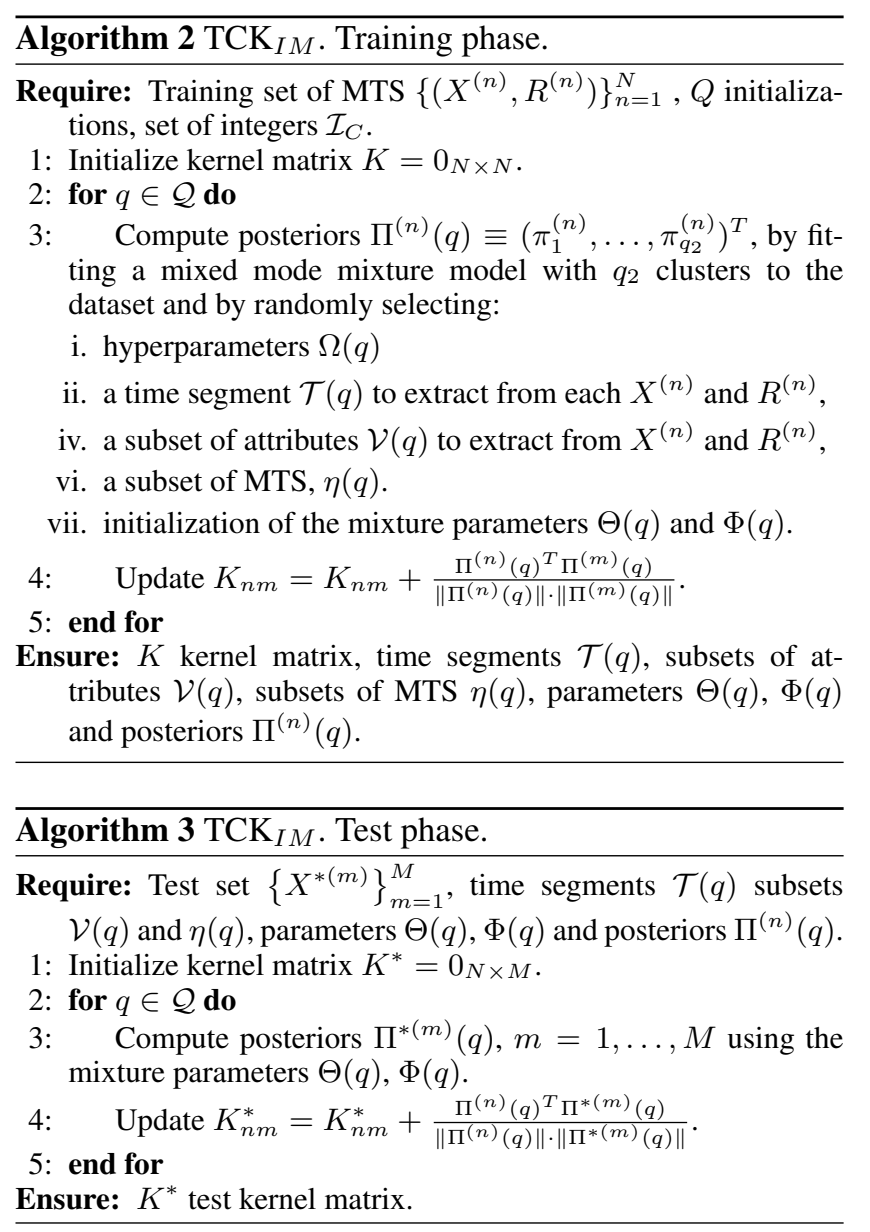

each $q$, we select random subsets of variables $\mathcal{V}(q)$ as well as random time segments $\mathcal{T}(q)$. After having trained the individual base models using an embarrasingly parallel procedure, we compute a normalized sum of the inner products of the normalized posterior distributions from each mixture component to build the $\mathrm{TCK}_{I M}$ kernel matrix. Details of the method are presented in Alg. 2, whereas Alg. 3 describes how to compute the kernel for MTS not seen during training.

\section{Experiments}

To test the performance of the proposed kernel, we considered three clinical datasets of which the characteristics are summarized in Tab. 1. The variables and the corresponding missing rates in the three datasets are summarized in Tab. 2 . A more detailed description of the datasets follows below.

Table 1: Description of the three real-world clinical datasets.

\begin{tabular}{l|lll}
\hline Dataset & PhysioNet & SSI & AL \\
\hline \# of patients & 4000 & 858 & 402 \\
\# attrib (Lab, vital) & $28(17,11)$ & $11(11,0)$ & $11(7,4)$ \\
Length of MTS & 48 (hours) & 10 (days) & 15 (days) \\
Positive class & $874(21.9 \%)$ & $227(26.5 \%)$ & $31(7.7 \%)$ \\
Av. missing rate & $76.6 \%$ & $80.7 \%$ & $83 \%$ \\
\hline
\end{tabular}

Table 2: List of variables and corresponding missing rates.

\begin{tabular}{|c|c|c|c|}
\hline & \multicolumn{3}{|c|}{ Variables (missing rate) } \\
\hline \multirow{9}{*}{ Phys } & Albumin (0.99) & $\operatorname{ALP}(0.98)$ & AST (0.98) \\
\hline & Bilirubin (0.98) & BUN (0.93) & Creat. (0.93) \\
\hline & Diast. BP $(0.11)$ & FiO2 (0.84) & $\operatorname{GCS}(0.68)$ \\
\hline & Glucose (0.93) & $\mathrm{HCO} 3(0.93)$ & HCT (0.91) \\
\hline & $\operatorname{HR}(0.10)$ & $\mathrm{K}(0.93)$ & Lactate $(0.96)$ \\
\hline & MAP (0.12) & $\operatorname{Mg}(0.93)$ & $\mathrm{Na}(0.93)$ \\
\hline & $\mathrm{PaCO} 2(0.88)$ & $\mathrm{PaO} 2(0.88)$ & Platelets (0.93) \\
\hline & RespRate (0.76) & $\mathrm{SaO} 2(0.96)$ & Syst. BP (0.11) \\
\hline & $\begin{array}{l}\text { Temp (0.63) } \\
\text { pH }(0.88)\end{array}$ & Urine $(0.31)$ & WBC (0.93) \\
\hline \multirow[t]{4}{*}{ SSI } & Albumin $(0.79)$ & Amylase (0.95) & Creat. (0.87) \\
\hline & CRP (0.69) & Glucose (0.92) & $\mathrm{Hb}(0.65)$ \\
\hline & $\mathrm{K}(0.71)$ & $\mathrm{Na}(0.71)$ & Platelets (0.92) \\
\hline & Urea $(0.94)$ & WBC (0.73) & \\
\hline \multirow[t]{4}{*}{$\mathrm{AL}$} & Albumin (0.80) & Creat. (0.94) & CRP (0.77) \\
\hline & Diast. BP (0.76) & $\mathrm{Hb}(0.71)$ & HR $(0.76)$ \\
\hline & $\mathrm{K}(0.75)$ & $\mathrm{Na}(0.75)$ & Syst. BP $(0.76)$ \\
\hline & Temp (0.66) & WBC $(0.80)$ & \\
\hline
\end{tabular}

PhysioNet The PhysioNet dataset is collected from the PhysioNet Challenge 2012 (Silva and others 2012). We extracted the first part, which consists of 4000 patient records of patients from the intensive care units (ICUs) of various hospitals. Each patient stayed in the ICU for at least 48 hours and the records contain information about both vital signs and blood samples collected over time. We extracted all measurements taken within 48 hours after admission and aligned the MTS into same-length sequences using an hourly discretization. Variables with a missing rate higher than $99 \%$ were omitted, which led to a total of 28 variables. The classification task was to predict whether the patient was recovering from surgery, which is a task that also has been considered in other work (Che and et al. 2018; Li and Xu 2019).

Surgical site infection This dataset contains data for 11 blood samples collected postoperatively for patients who underwent major abdominal surgery at the department of gastrointestinal surgery at a Norwegian university hospital in the years 2004-2012. The task considered was to detect surgical site infection (SSI), which is one of the most common types of nosocomial infections (Lewis and others 2013) and represents up to $30 \%$ of all hospital-acquired infections (Magill and others 2012). Patients with no recorded lab tests during the period from postoperative day 1 until day 10 were removed from the cohort, which lead to a final cohort consisting of 858 patients. The average proportion of missing data in the cohort was $80.7 \%$. To identify the patients in the cohort who developed postoperative SSI and create ground truth labels, ICD-10 as well as NOMESCO Classification of Surgical Procedures codes related to severe postoperative complications were considered. Patients without these codes who also did not have a mention of the word "infection" in any of their postoperative text documents were considered as controls. This lead to a dataset consisting of 227 infected patients and 631 non-infected patients.

Anastomosis leakage Anastomosis leakage (AL) is potentially a serious complication that can occur after colon 
rectal cancer (CRC) surgery, of which one of the consequences is an increase in 30-day mortality (Snijders and others 2013). It is estimated that 5-15\% of the patients who undergo surgery for CRC suffer from AL (Branagan and Finnis 2005). Recent studies have shown that both unstructured as well structured EHR data such as measurements of blood tests and vital signs could have predictive value for AL (Soguero-Ruiz and others 2016; Soguero-Ruiz et al. 2014). The dataset considered in this work contains only structured data and is collected from the same hospital as the SSI dataset. It contains physiological data (blood tests and vital signs) for the period from the day after surgery to day 15 for 402 patients who underwent CRC surgery. A total of 31 of these patients got AL after surgery, but there is no information available about exactly when it happened. The classification task considered here was to detect which of the patients got AL.

Experimental setup We considered the following experimental setup. We performed kernel principal component analysis (KPCA) (Schölkopf, Smola, and Müller 1997) using the proposed $\mathrm{TCK}_{I M}$ and then trained a $\mathrm{kNN}$-classifier in the low dimensional space. The dimensionality of the KPCA-representation was set to 3 to also be able to visualize the embeddings, whereas we used 5-fold cross validation to set the number of neighbors $k$ for the $\mathrm{kNN}$-classifier. Performance was measured in terms of F1-score, sensitivity and specificity. Sensitivity is the fraction of correctly classified cases, whereas specificity is the fraction of controls that are correctly classified as negative. F1-score is the harmonic mean of precision and sensitivity, where precision is the fraction of actual positives among all those that are classified as positive cases.

We compared the performance of the proposed kernel to four baseline kernels, namely the linear kernel (Lin), the global alignment kernel (GAK) (Cuturi 2011b), LPS and TCK. GAK is a positive semi-definite kernel formulation of the widely used, but non-metric, time series similarity measure called dynamic time warping (DTW) (Berndt and Clifford 1994). It has two hyperparameters, namely the kernel bandwidth and the triangular parameter, which have to be set by the user and it does not naturally deal with missing data and incomplete datasets, and therefore also requires a preprocessing step involving imputation. Therefore, we created a complete dataset using mean imputation for Lin and GAK (initial experiments showed that mean imputation worked better than last observation carried forward). In accordance with (Cuturi 2011a), for GAK we set the bandwidth $\sigma$ to 0.1 times the median distance of all MTS in the training set scaled by the square root of the median length of all MTS, and the triangular parameter to 0.2 times the median length (Frobenius norm) of all MTS. In order to design baseline kernels that can exploit informative missingness, we also created baselines (referred to as $\operatorname{Lin}_{I M}, \mathrm{GAK}_{I M}$ and $\mathrm{LPS}_{I M}$ ) by concatenating the binary indicator MTS $R^{(n)}$ to $X^{(n)}$. LPS was run with default hyperparameters using the implementation provided by (Baydogan 2014), with the exception that the minimal
Table 3: Performance (mean \pm se) on 3 datasets.

\begin{tabular}{|c|c|c|c|c|}
\hline & Kernel & Sensitivity & Specificitv & F1-score \\
\hline \multirow{8}{*}{ Phys. } & Lin & $0.329 \pm 0.049$ & $0.812 \pm 0.007$ & $0.328 \pm 0.052$ \\
\hline & GAK & $0.313 \pm 0.021$ & $0.801 \pm 0.019$ & $0.309 \pm 0.012$ \\
\hline & LPS & $0.511 \pm 0.069$ & $0.948 \pm 0.010$ & $0.600 \pm 0.068$ \\
\hline & TCK & $0.411 \pm 0.053$ & $0.833 \pm 0.022$ & $0.408 \pm 0.049$ \\
\hline & $\operatorname{Lin}_{I M}$ & $0.556 \pm 0.054$ & $0.939 \pm 0.013$ & $0.625 \pm 0.046$ \\
\hline & $\mathrm{GAK}_{I M}$ & $0.566 \pm 0.032$ & $0.941 \pm 0.010$ & $0.636 \pm 0.038$ \\
\hline & $\operatorname{LPS}_{I M}$ & $0.611 \pm 0.060$ & $0.939 \pm 0.009$ & $0.667 \pm 0.041$ \\
\hline & $\mathrm{TCK}_{I M}$ & $\mathbf{0 . 6 9 9} \pm \mathbf{0 . 0 3 4}$ & $\mathbf{0 . 9 8 0} \pm \mathbf{0 . 0 0 7}$ & $\mathbf{0 . 7 8 9} \pm \mathbf{0 . 0 3 3}$ \\
\hline \multirow{8}{*}{ SSI } & Lin & $0.480 \pm 0.041$ & $0.878 \pm 0.041$ & $0.529 \pm 0.072$ \\
\hline & GAK & $0.639 \pm 0.064$ & $0.921 \pm 0.036$ & $0.687 \pm 0.025$ \\
\hline & LPS & $0.687 \pm 0.044$ & $0.929 \pm 0.040$ & $0.730 \pm 0.028$ \\
\hline & TCK & $0.683 \pm 0.071$ & $0.922 \pm 0.025$ & $0.719 \pm 0.069$ \\
\hline & $\operatorname{Lin}_{I M}$ & $0.700 \pm 0.012$ & $\mathbf{0 . 9 4 4} \pm \mathbf{0 . 0 3 0}$ & $0.755 \pm 0.024$ \\
\hline & $\mathrm{GAK}_{I M}$ & $0.718 \pm 0.073$ & $0.940 \pm 0.026$ & $0.761 \pm 0.036$ \\
\hline & $\operatorname{LPS}_{I M}$ & $0.652 \pm 0.056$ & $0.925 \pm 0.015$ & $0.701 \pm 0.036$ \\
\hline & $\mathrm{TCK}_{I M}$ & $\mathbf{0 . 7 7 5} \pm \mathbf{0 . 0 1 7}$ & $0.929 \pm 0.010$ & $\mathbf{0 . 7 8 6} \pm \mathbf{0 . 0 1 5}$ \\
\hline \multirow{8}{*}{ AL } & Lin & $0.414 \pm 0.273$ & $0.907 \pm 0.119$ & $0.468 \pm 0.240$ \\
\hline & GAK & $0.428 \pm 0.223$ & $\mathbf{0 . 9 3 5} \pm \mathbf{0 . 0 7 8}$ & $0.520 \pm 0.206$ \\
\hline & LPS & $\mathbf{0 . 8 4 3} \pm \mathbf{0 . 1 0 5}$ & $0.835 \pm 0.075$ & $0.776 \pm 0.058$ \\
\hline & TCK & $0.700 \pm 0.147$ & $0.886 \pm 0.107$ & $0.722 \pm 0.080$ \\
\hline & $\operatorname{Lin}_{I M}$ & $0.742 \pm 0.221$ & $0.864 \pm 0.097$ & $0.724 \pm 0.097$ \\
\hline & $\mathrm{GAK}_{I M}$ & $0.728 \pm 0.227$ & $0.928 \pm 0.047$ & $0.759 \pm 0.156$ \\
\hline & $\operatorname{LPS}_{I M}$ & $0.800 \pm 0.120$ & $0.864 \pm 0.091$ & $0.773 \pm 0.091$ \\
\hline & $\mathrm{TCK}_{I M}$ & $\mathbf{0 . 8 4 3} \pm \mathbf{0 . 1 8 4}$ & $0.864 \pm 0.095$ & $\mathbf{0 . 7 9 2} \pm \mathbf{0 . 1 1 5}$ \\
\hline
\end{tabular}

segment length was adjusted to account for the relatively short MTS in the datasets. For the $\mathrm{TCK}_{I M}$ we let $Q=15$ and $\mathcal{I}_{C}=\{N / 200, \ldots, N / 200+20\}$, and, likewise, TCK was run with $Q=15$ and $C=20$. For all methods, except LPS which do not require standardization, we standardized each attribute to zero mean and unit standard deviation.

For PhysioNet and SSI, we did 5-fold cross validation to measure performance. The AL dataset is, however, highly imbalanced and therefore we employed an undersampling strategy by randomly sampling two patients from the negative class per positive case. $20 \%$ of this dataset was then set aside as a test set. This entire process was repeated 10 times and we reported mean and standard errors of the performance measures. The AL dataset is small, and for that reason the hyperparameters of the methods had to be adjusted accordingly. For $\mathrm{TCK}_{I M}$ we let $Q=10$ and $\mathcal{I}_{C}=\{2,3\}$.

\section{Results and discussion}

Tab. 3 shows the performance of the $\mathrm{TCK}_{I M}$ kernel, as well as the baseline methods, on the three real-world datasets. The first thing to notice is that the two kernels (Lin and GAK) that rely on imputation consistently perform much worse than the other kernels in terms of both F1-score across all datasets. We also note that these methods achieve a relatively high specificity. However, this is because they put too many patients in the negative class, which also leads to a high false negative rate and, consequently, a low sensitivity. The reasons could be that the imputation methods introduce biases and that the missingness mechanism is ignored.

The two kernels, TCK and LPS, that naturally handle the missing data perform better than the kernels that rely on imputation for all three datasets. TCK and LPS perform quite 

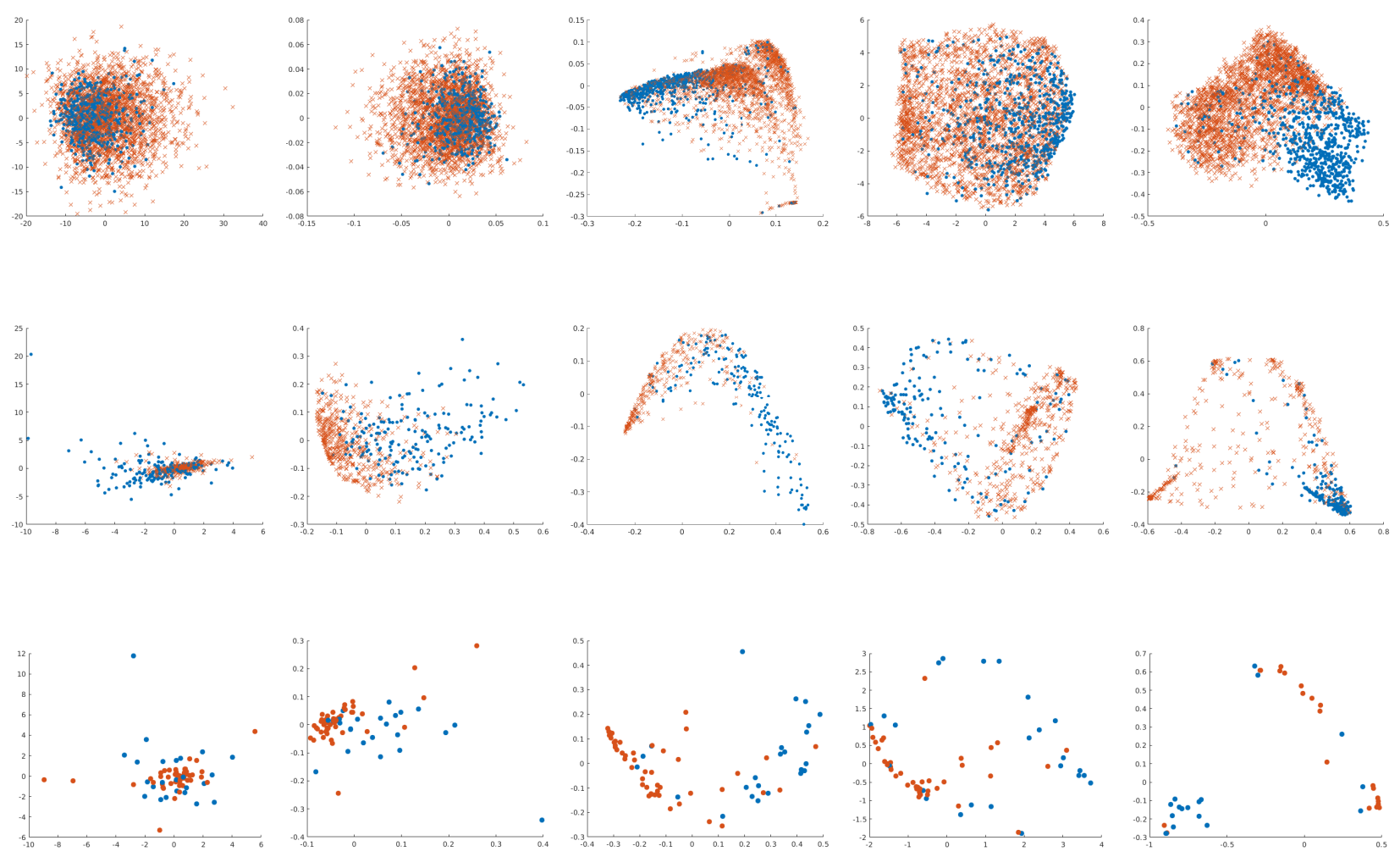

Figure 1: KPCA plots obtained using Lin, GAK, LPS, TCK and TCK $I M$, respectively. Row 1: Physio., row 2: SSI, row 3: AL.

similarly across all 3 evaluation metrics for the SSI dataset, whereas LPS outperforms TCK on the PhysioNet and AL dataset. These methods probably perform better than the imputation methods because ignoring the missingness introduces less bias than replacing missing values with biased estimates. The performance of the baselines that account for informative missingness, $\operatorname{Lin}_{I M}$ and $\mathrm{GAK}_{I M}$, is considerably better than Lin and GAK, respectively, for all datasets. LPS $_{I M}$ also performs better than LPS on the PhysioNet datasets, whereas the performance of these two baselines is more or less equal on the two other datasets. The proposed $\mathrm{TCK}_{I M}$ performs considerably better than all baselines, and in particular compared to TCK (the kernel which it is an improvement of) for the PhysioNet and SSI datasets in terms of F1-score, and it performs better or comparable than the other kernels on the AL dataset. This demonstrates that the missing patterns in clinical time series are often informative and the $\mathrm{TCK}_{I M}$ can exploit this information very efficiently.

Fig. 11 shows the KPCA embeddings obtained using five kernels (Lin, GAK, LPS, TCK and TCK $I M$ ). In general, the classes are more separated in the representations obtained using $\mathrm{TCK}_{I M}$ than in the other representations.

Limitations, future work and conclusions In this paper, we presented a MTS kernel capable of exploiting informative missingness along with the temporal dependencies in the observed data. We showed that $\mathrm{TCK}_{I M}$ can learn good representations that can be exploited both in supervised and unsupervised tasks, even when the percentage of missing data is high. In this work, the representations learned using TCK $_{I M}$ were evaluated visually (Fig. 1) and using a supervised scheme by training a classifier (Tab. 3). The experimental results suggested that $\mathrm{TCK}_{I M}$ achieved superior performances compared to baselines on three real datasets.

The experiments presented in this work focused on binary classification tasks, both of patients at the ICU and patients who had undergone colonrectal cancer surgery. However, we believe that $\mathrm{TCK}_{I M}$ is also a very good choice in applications where there is a lack of labels, which often is the case in medical applications, thanks to the ensemble learning strategy that makes the kernel robust to hyperparameter choices. In fact, since it is a kernel, it can be used in many different applications, including classification as well as clustering tasks, benefiting from the vast body of work in the field of kernel methods. In future work, we would like to test $\mathrm{TCK}_{I M}$ in a realistic unsupervised task from the medical domain.

A limitation of $\mathrm{TCK}_{I M}$ is that it is only designed for MTS of the same length. In further work, we would therefore like to design a time series cluster kernel that can also deal with varying length MTS. It should also be pointed out that if the missing patterns are not informative, i.e. the missingness is not correlated the particular medical condition(s) of interest, the performance gain of $\mathrm{TCK}_{I M}$ compared to TCK is low. It is therefore an advantage if the user has some understanding about the underlying missingness mechanism in 
the data. On the other hand, our experiments on benchmark datasets (see Appendix) demonstrate that in cases when the missingness mechanism is almost ignorable (low correlation between missing patterns and labels), the performance of $\mathrm{TCK}_{I M}$ is not worse than TCK.

\section{Acknowledgement}

The authors would like to thank K. Hindberg for assistance on extraction of EHR data and the physicians A. Revhaug, R.-O. Lindsetmo and K. M. Augestad for helpful guidance throughout the study.

\section{Appendix - Synthetic benchmark datasets}

To test how well $\mathrm{TCK}_{I M}$ performs for a varying degree of informative missingness, we generated in total 16 synthetic datasets by randomly injecting missing data into 4 MTS benchmark datasets. The characteristics of the datasets are described in Tab. 4 We transformed all MTS in each dataset to the same length, $T$, where $\mathrm{T}$ is given by $T=$ $\left\lceil T_{\max } /\left\lceil T_{\max } / 25\right\rceil\right\rceil$. Here, \lceil\rceil is the ceiling operator and $T_{\max }$ is the length of the longest MTS in the original dataset.

Table 4: Characteristics of the benchmark datasets. Attr is the number of attributes, Train and Test the number of training and test samples. $N_{c}$ is the number of classes, $T_{\min }$ and $T_{\max }$ the length of shortest and longest MTS in the dataset, whereas $T$ is the length of the MTS after the transformation. Datasets Attr Train Test $N_{c} T_{\min } T_{\max } T \quad$ Source \begin{tabular}{llllllll}
\hline uWave & 3 & 200 & 4278 & 8 & 315 & 315 & 25 (Bagnall and others 2018)
\end{tabular} Char.tra. $\quad 3 \quad 300 \quad 2558 \quad 20 \quad 109 \quad 205 \quad 23$ (Bagnall and others 2018) $\begin{array}{lllllllll}\text { Wafer } & 6 & 298 & 896 & 2 & 104 & 198 & 25 & \text { (Olszewsk1 2001) }\end{array}$ Jap.vow. $12 \quad 270 \quad 370 \quad 9 \quad 7 \quad 29 \quad 15$ (Bagnall and others 2018)

Datasets The following procedure was used to create 8 synthetic datasets with missing data from the Wafer and Japanese vowels datasets. We randomly sampled a number $c_{v} \in\{-1,1\}$ for each attribute $v \in\{1, \ldots, V\}$, where $c_{v}=1$ indicates that the attribute and the labels are positively correlated and $c_{v}=-1$ negatively correlated. Thereafter, we sampled a missing rate $\gamma_{n v}$ from $\mathcal{U}[0.3+E$. $\left.c_{v} \cdot\left(y^{(n)}-1\right), 0.7+E \cdot c_{v} \cdot\left(y^{(n)}-1\right)\right]$ for each MTS $X^{(n)}$ and attribute. The parameter $E$ was tuned such that the Pearson correlation (absolute value) between the missing rates for the attributes $\gamma_{v}$ and the labels $y^{(n)}$ took the values $\{0.2,0.4,0.6,0.8\}$, respectively. By doing so, we could control the amount of informative missingness and because of the way we sampled $\gamma_{n v}$, the missing rate in each dataset was around $50 \%$ independently of the Pearson correlation.

Further, the following procedure was used to create 8 synthetic datasets from the uWave and Character trajectories datasets, which both consist of only 3 attributes. We randomly sampled a number $c_{v} \in\{-1,1\}$ for each attribute $v \in\{1, \ldots, V\}$. Attribute(s) with $c_{v}=-1$ became negatively correlated with the labels by sampling $\gamma_{n v}$ from $\mathcal{U}\left[0.7-E \cdot\left(y^{(n)}-1\right), 1-E \cdot\left(y^{(n)}-1\right)\right]$, whereas the attribute(s) with $c_{v}=1$ became positively correlated with the labels by sampling $\gamma_{n v}$ from $\mathcal{U}\left[0.3+E \cdot\left(y^{(n)}-1\right), 0.6+\right.$ $\left.E \cdot\left(y^{(n)}-1\right)\right]$. The parameter $E$ was computed in the same way as above. Then, we computed the mean of each attribute $\mu_{v}$ over the complete dataset and let each element with $x_{v}^{(n)}(t)>\mu_{v}$ be missing with probability $\gamma_{n v}$. This means that the probability of being missing is dependent on the value of the missing element, i.e. the missingness mechanism is MNAR within each class. Hence, this type of informative missingness is not the same as the one we created for the Wafer and Japanese vowels datasets.

Baselines Three baseline models were created. The first baseline, namely ordinary TCK, ignores the missingness mechanism. We created a second baseline, refered to as $\mathrm{TCK}_{B}$, in which the missing patterns we modeled naively by concatenating the binary missing indicator MTS $R$ to the MTS $X$ and creating a new MTS $U$ with $2 V$ attributes. Then, ordinary TCK was trained on the datasets consisting of $\left\{U^{(n)}\right\}$. In the third baseline, $\mathrm{TCK}_{0}$, we investigated how well informative missingness can be captured by imputing zeros for the missing values and then training the TCK on the imputed data.

Table 5: Performance (accuracy) on the 16 synthetic datasets

\begin{tabular}{|l|l|llll|}
\hline Dataset & Corr. & TCK & TCK $_{B}$ & TCK $_{0}$ & TCK $_{I M}$ \\
\hline \multirow{4}{*}{ Wafer } & 0.2 & 0.951 & 0.951 & 0.951 & $\mathbf{0 . 9 5 5}$ \\
& 0.4 & $\mathbf{0 . 9 6 1}$ & 0.953 & 0.955 & $\mathbf{0 . 9 6 1}$ \\
& 0.6 & 0.961 & 0.900 & 0.965 & $\mathbf{0 . 9 9 6}$ \\
& 0.8 & 0.958 & 0.893 & 0.963 & $\mathbf{1 . 0 0 0}$ \\
\hline \multirow{4}{*}{ Japan. vow. } & 0.2 & 0.938 & $\mathbf{0 . 9 5 4}$ & 0.951 & 0.940 \\
& 0.4 & 0.932 & 0.938 & 0.938 & $\mathbf{0 . 9 4 1}$ \\
& 0.6 & 0.922 & 0.946 & 0.924 & $\mathbf{0 . 9 6 2}$ \\
& 0.8 & 0.922 & 0.924 & 0.935 & $\mathbf{0 . 9 6 8}$ \\
\hline \multirow{4}{*}{ uWave } & 0.2 & 0.763 & 0.457 & 0.755 & $\mathbf{0 . 8 4 1}$ \\
& 0.4 & 0.807 & 0.587 & 0.813 & $\mathbf{0 . 8 5 7}$ \\
& 0.6 & 0.831 & 0.674 & 0.837 & $\mathbf{0 . 8 6 5}$ \\
& 0.8 & 0.834 & 0.699 & 0.844 & $\mathbf{0 . 8 8 4}$ \\
\hline \multirow{3}{*}{ Char. Traj. } & 0.2 & $\mathbf{0 . 8 5 4}$ & 0.742 & 0.847 & 0.851 \\
& 0.4 & 0.851 & 0.788 & 0.842 & $\mathbf{0 . 8 6 7}$ \\
& 0.6 & 0.825 & 0.790 & 0.824 & $\mathbf{0 . 8 7 1}$ \\
& 0.8 & 0.839 & 0.707 & 0.853 & $\mathbf{0 . 9 0 1}$ \\
\hline
\end{tabular}

Results Tab. 5 shows the performance of the proposed $\mathrm{TCK}_{I M}$ and the three baselines for all of the 16 synthetic datasets. We see that the proposed $\mathrm{TCK}_{I M}$ achieves the best accuracy for 14 out of 16 datasets and is the only method which consistently has the expected behaviour, namely that the accuracy increases as the correlation between missing values and class labels increases. It can also be seen that the performance of $\mathrm{TCK}_{I M}$ is similar to TCK when the amount of information in the missing patterns is low, whereas TCK is clearly outperformed when the informative missingness is high. This demonstrates that $\mathrm{TCK}_{I M}$ can effectively exploit informative missingness. 


\section{References}

[Agniel and others 2018] Agniel, D., et al. 2018. Biases in electronic health record data due to processes within the healthcare system: retrospective observational study. BMJ 361:k1479.

[Bagnall and others 2018] Bagnall, A., et al. 2018. The uea multivariate time series classification archive, 2018. arXiv preprint arXiv:1811.00075.

[Baydogan and Runger 2016] Baydogan, M. G., and Runger, G. 2016. Time series representation and similarity based on local autopatterns. Data Mining and Knowledge Discovery 30(2):476-509.

[Baydogan 2014] Baydogan, M. 2014. LPS Matlab implementation. http://www.mustafabaydogan.com/ Accessed: 2019-09-06.

[Berndt and Clifford 1994] Berndt, D. J., and Clifford, J. 1994. Using dynamic time warping to find patterns in time series. In $3 r d$ Int Conf Knowl Discovery and Data Mining, 359-370. AAAI Press.

[Bianchi and others 2019] Bianchi, F. M., et al. 2019. Learning representations of multivariate time series with missing data. Patt. Rec. 96:106973.

[Bianchi et al. 2018] Bianchi, F. M.; Livi, L.; Ferrante, A.; et al. 2018. Time series kernel similarities for predicting paroxysmal atrial fibrillation from ECGs. In IJCNN, 1-8.

[Branagan and Finnis 2005] Branagan, G., and Finnis, D. 2005. Prognosis after anastomotic leakage in colorectal surgery. Dis Colon Rectum 48(5):1021-1026.

[Che and et al. 2018] Che, Z., and et al. 2018. Recurrent neural networks for multivariate time series with missing values. Scientific reports 8(1):6085.

[Cuturi 2011a] Cuturi, M. 2011a. Fast global alignment kernel Matlab implementation. http://www.marcocuturi.net/GA.html Accessed: 2019-09-02.

[Cuturi 2011b] Cuturi, M. 2011b. Fast global alignment kernels. In Proc. 28th Int. Conf. on Machine Learning, 929-936.

[Dempster, Laird, and Rubin 1977] Dempster, A. P.; Laird, N. M.; and Rubin, D. B. 1977. Maximum likelihood from incomplete data via the EM algorithm. Jour. royal stat.l society. Ser. B (methodological) $1-38$.

[Dietterich 2000] Dietterich, T. G. 2000. Ensemble methods in machine learning. In Int workshop on multiple classifier systems, 115 .

[Donders and others 2006] Donders, A. R., et al. 2006. Review: a gentle introduction to imputation of missing values. J clin epidemiol 59(10):1087-1091.

[Ghassemi and others 2015] Ghassemi, M., et al. 2015. A multivariate timeseries modeling approach to severity of illness assessment and forecasting in ICU with sparse, heterogeneous clinical data. In Proc Conf AAAI Artif Intell, 446-453.

[Ghorbani and Zou 2018] Ghorbani, A., and Zou, J. Y. 2018. Embedding for informative missingness: Deep learning with incomplete data. In Conf Comm., Control, and Computing, 437-445. IEEE.

[Halpern and others 2016] Halpern, Y., et al. 2016. Electronic medical record phenotyping using the anchor and learn framework. $J$ Am Med Inform Assoc.

[Hansen and Salamon 1990] Hansen, L. K., and Salamon, P. 1990. Neural network ensembles. IEEE TPAMI 12(10):993-1001.

[Lewis and others 2013] Lewis, S. S., et al. 2013. Assessing the relative burden of hospital-acquired infections in a network of community hospitals. Infect Control Hosp Epidemiol. 34(11):12291230.
[Li and Xu 2019] Li, Q., and Xu, Y. 2019. VS-GRU: A variable sensitive gated recurrent neural network for multivariate time series with massive missing values. Applied Sciences 9(15):3041.

[Lipton, Kale, and Wetzel 2016] Lipton, Z. C.; Kale, D.; and Wetzel, R. 2016. Directly modeling missing data in sequences with RNNs: Improved classification of clinical time series. In Machine Learning for Healthcare Conference, volume 56, 253-270. PMLR.

[Little and Rubin 2014] Little, R. J., and Rubin, D. B. 2014. Statistical analysis with missing data. John Wiley \& Sons.

[Ma and Chen 2018] Ma, Z., and Chen, G. 2018. Bayesian methods for dealing with missing data problems. J Korean Stat Soc 47(3):297-313.

[Magill and others 2012] Magill, S. S., et al. 2012. Prevalence of healthcare-associated infections in acute care hospitals in Jacksonville, Florida. Infection Control 33(03):283-291.

[Mikalsen and others 2018] Mikalsen, K. Ø., et al. 2018. Time series cluster kernel for learning similarities between multivariate time series with missing data. Pattern Recognition 76:569-581.

[Mikalsen et al. 2016] Mikalsen, K. Ø.; Bianchi, F. M.; SogueroRuiz, C.; et al. 2016. Learning similarities between irregularly sampled short multivariate time series from EHRs. 3rd ICPR Int. Workshop on Patt. Rec. for Healthcare Analytics.

[Mikalsen et al. 2017] Mikalsen, K. Ø.; Soguero-Ruiz, C.; Revhaug, A.; Lindsetmo, R.-O.; Jenssen, R.; et al. 2017. Using anchors from free text in electronic health records to diagnose postoperative delirium. Computer Methods and Programs in Biomedicine 152:105 - 114

[Olszewski 2001] Olszewski, R. T. 2001. Generalized Feature Extraction for Structural Pattern Recognition in Time-series Data. Ph.D. Dissertation, Carnegie Mellon University, Pittsburgh, PA, USA.

[Rubin 1976] Rubin, D. B. $1976 . \quad$ Inference and missing data. Biometrika 63(3):581-592.

[Schafer and Graham 2002] Schafer, J. L., and Graham, J. W. 2002. Missing data: our view of the state of the art. Psychological methods 7(2): 147 .

[Schölkopf, Smola, and Müller 1997] Schölkopf, B.; Smola, A.; and Müller, K.-R. 1997. Kernel principal component analysis. In International Conference on Artificial Neural Networks, 583-588. Springer.

[Shao and Zhong 2003] Shao, J., and Zhong, B. 2003. Last observation carry-forward and last observation analysis. Statistics in medicine 22(15):2429-2441.

[Sharafoddini and others 2019] Sharafoddini, A., et al. 2019. A new insight into missing data in intensive care unit patient profiles: Observational study. JMIR Med Inform. 7(1):e11605.

[Shukla and Marlin 2019] Shukla, S. N., and Marlin, B. 2019. Interpolation-prediction networks for irregularly sampled time series. In $I C L R$.

[Silva and others 2012] Silva, I., et al. 2012. Predicting in-hospital mortality of ICU patients: The physionet/computing in cardiology challenge 2012. In 2012 Computing in Cardiology, 245-248. IEEE.

[Snijders and others 2013] Snijders, H., et al. 2013. Anastomotic leakage as an outcome measure for quality of colorectal cancer surgery. BMJ Qual Saf 22(9):759-767.

[Soguero-Ruiz and others 2016] Soguero-Ruiz, C., et al. 2016. Predicting colorectal surgical complications using heterogeneous clinical data and kernel methods. J Biomed Inform. 61:87-96. 
[Soguero-Ruiz et al. 2014] Soguero-Ruiz, C.; Hindberg, K.; Jenssen, R.; et al. 2014. Support vector feature selection for early detection of anastomosis leakage from bag-of-words in electronic health records. IEEE J Biomed Health Inform. 20(5):1404-1415.

[Wells et al. 2013] Wells, B. J.; Chagin, K. M.; Nowacki, A. S.; and Kattan, M. W. 2013. Strategies for handling missing data in electronic health record derived data. eGEMs 1(3).

[Zhang 2016] Zhang, Z. 2016. Missing data imputation: focusing on single imputation. Ann Transl Med 4(1). 